\title{
ZEUS AT HERA II
}

\author{
B. FOSTER \\ H.H. Wills Physics Lab., Tyndall Avenue, Bristol, BS8 1TL, U.K. \\ E-mail: b.foster@bris.ac.uk
}

\begin{abstract}
The ZEUS detector has been upgraded in a number of areas to prepare for the physics opportunities of HERA II. These upgrades, and their physics rationale and promise, are briefly outlined. The measurement of polarisation at HERA II, and its importance for the HERA II physics programme, is also discussed.
\end{abstract}

\section{Introduction}

The HERA upgrade will produce about a factor five improvement in luminosity delivered to the experiments and aims to accumulate $1 \mathrm{fb}^{-1}$ of data in the HERA II programme. In order to take advantage of this, the ZEUS detector has been upgraded in several areas: the silicon microvertex detector (MVD); the straw-tube tracker (STT); and the luminosity monitor. The addition of spin rotators will produce longitudinal polarisation in both ZEUS and H1; both experiments have collaborated with HERMES and HERA to produce an accurate measurement of the degree of polarisation.

This talk reviews the characteristics and status of the major upgrades, together with the physics towards which they are aimed. It is not a comprehensive review of HERA II physics, and should be read in conjunction with the talks at this workshop by E. Elsen, which covers complementary areas, and by W. Buchmüller.

\section{The vertex region}

The ZEUS MVD 1 consists of $20 \mu \mathrm{m}$ pitch $n$-type silicon-strip detectors with $p^{+}$-type implants. The readout pitch is $120 \mu \mathrm{m}$, leading to more than 200,000 readout channels, which are digitised by a custom-built clock, control and ADC system. The detectors are organised in two main groups: a "barrel", which surrounds the elliptical $2 \mathrm{~mm}$-thick ( $\sim 1.1 \%$ of a radiation length) aluminium-beryllium beam-pipe; and four "wheels", consisting of wedgeshaped detectors mounted perpendicular to the beam-line and displaced in the proton, or "forward" direction. The layout of the barrel detector is shown in Fig. 11a); it gives maximum coverage for charged particles emanating from the interaction point while accommodating the elliptical beam-pipe. Most tracks pass through three separate detectors as they leave the interaction

DIS01 LANL: submitted to World Scientific on 8th November 2018 

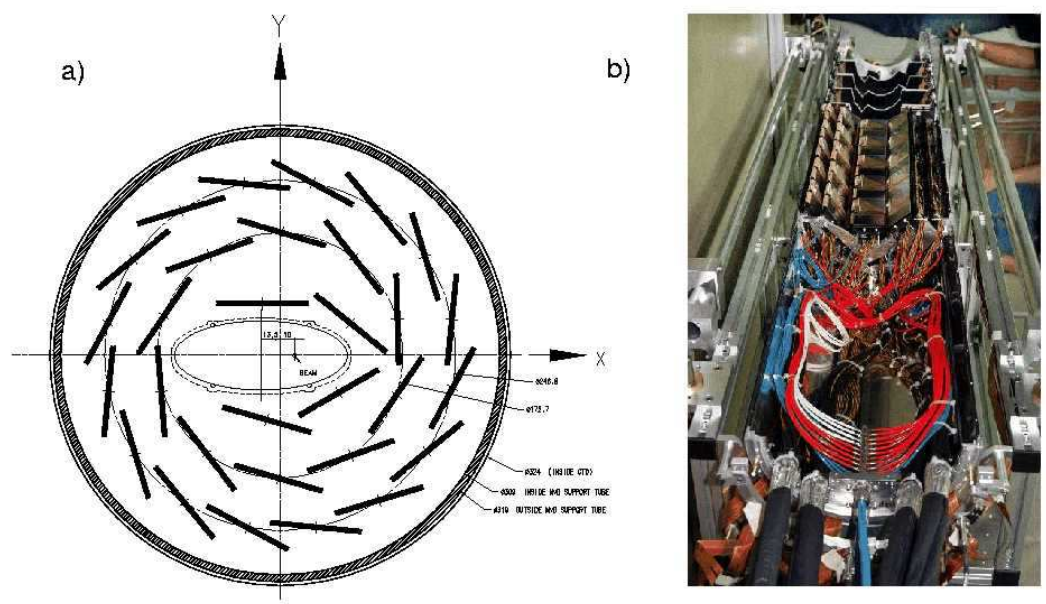

Figure 1. a) A section through the barrel MVD, showing the arrangement around the beampipe of each of the MVD ladders. b) A photograph of one half of the MVD, showing the barrel ladders, one half of each of the four forward "wheels" and the cables and services.

point; the remainder pass through two. Figure $1 \mathrm{~b}$ ) shows one half of the MVD before installation at DESY. In the barrel region, the ladders, each of which consists of 5 silicon detectors, and halves of the four forward "wheels", can be seen, as can the dense array of readout and services cables and the cooling system. The complete MVD was installed in ZEUS in April 2001.

The physics programme addressed by the MVD is that of the flavour decomposition of the proton and photon and the search for physics beyond the Standard Model. At HERA I, precise measurements of the inclusive structure function, $F_{2}$, have been made over a large kinematic range. In addition, measurements of the semi-inclusive charm structure function, $F_{2}^{c}$, have also been made, but these are limited by statistics. The large increase in luminosity of HERA II, together with the ability to tag heavy-quark decays in the MVD, should improved the measurement of $F_{2}^{c}$. After about $500 \mathrm{pb}^{-1}$, an uncertainty of around the $2 \%$ currently measured on $F_{2}$ should be obtained. In addition $b$-quark production can be measured precisely; a Monte Carlo simulation 1 of a measurement of $F_{2}^{b} / F_{2}^{c}$ after $500 \mathrm{pb}^{-1}$ is shown in Figure 2 .

It should also be possible, from a combination on neutral and charged current measurements, to separate out the $u, d, s, c, b$ and $g$ contribution to $F_{2}$. The MVD will not only be vital in the extraction of the $b$ and $c$ signal; it will also be important to measure the strange quark contribution $\mathrm{B}$, by 


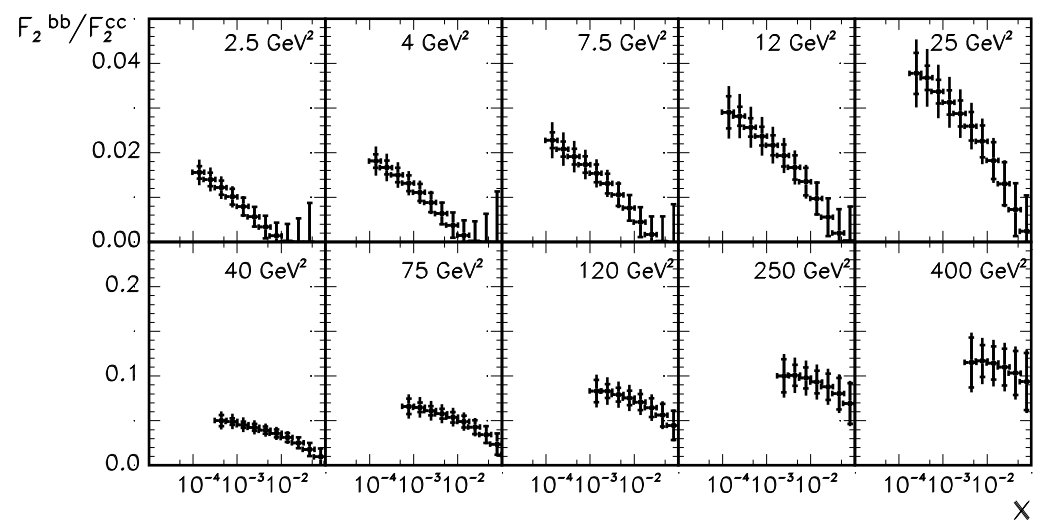

Figure 2. The ratio of the contribution of $b$-quark to $c$-quark production in $Q^{2}$ bins as a function of Bjorken $x$.

looking for charm in charged current interactions via the transition $s \stackrel{W}{\rightarrow} c$. Since many possible exotic particles tend to decay preferentially to heavy quarks, the MVD will also help in searches for physics beyond the Standard Model.

\section{Charged-particle tracking in the forward direction}

The higher luminosity expected at HERA II will increase the number of very high $Q^{2}$ events in which the electron or positron is scattered into the forward direction. It will also give access to rare processes, including possible physics beyond the Standard Model, which tend to have forward jets. The patternrecognition capabilities of the ZEUS Forward Tracker have therefore been improved by the replacement of two layers of transition radiation detector by layers of straw tubes, as shown schematically in Fig. 3. The straws are approximately $7.5 \mathrm{~mm}$ in diameter and range in length from around $20 \mathrm{~cm}$ to just over $1 \mathrm{~m}$. They are constructed from two layers of $50 \mu \mathrm{m}$ kapton foil coated with a $0.2 \mu \mathrm{m}$ layer of aluminium, surrounding a $50 \mu \mathrm{m}$ wire at the centre. The straws are arranged in wedges consisting of three layers rotated with respect to each other to give three-dimensional reconstruction. Each of the two 'supermodules' consists of four layers of such wedges. Figure 3 shows, schematically, the arrangement of the new forward detector, together with the central tracking detector (CTD) and the MVD. The smaller of the two 


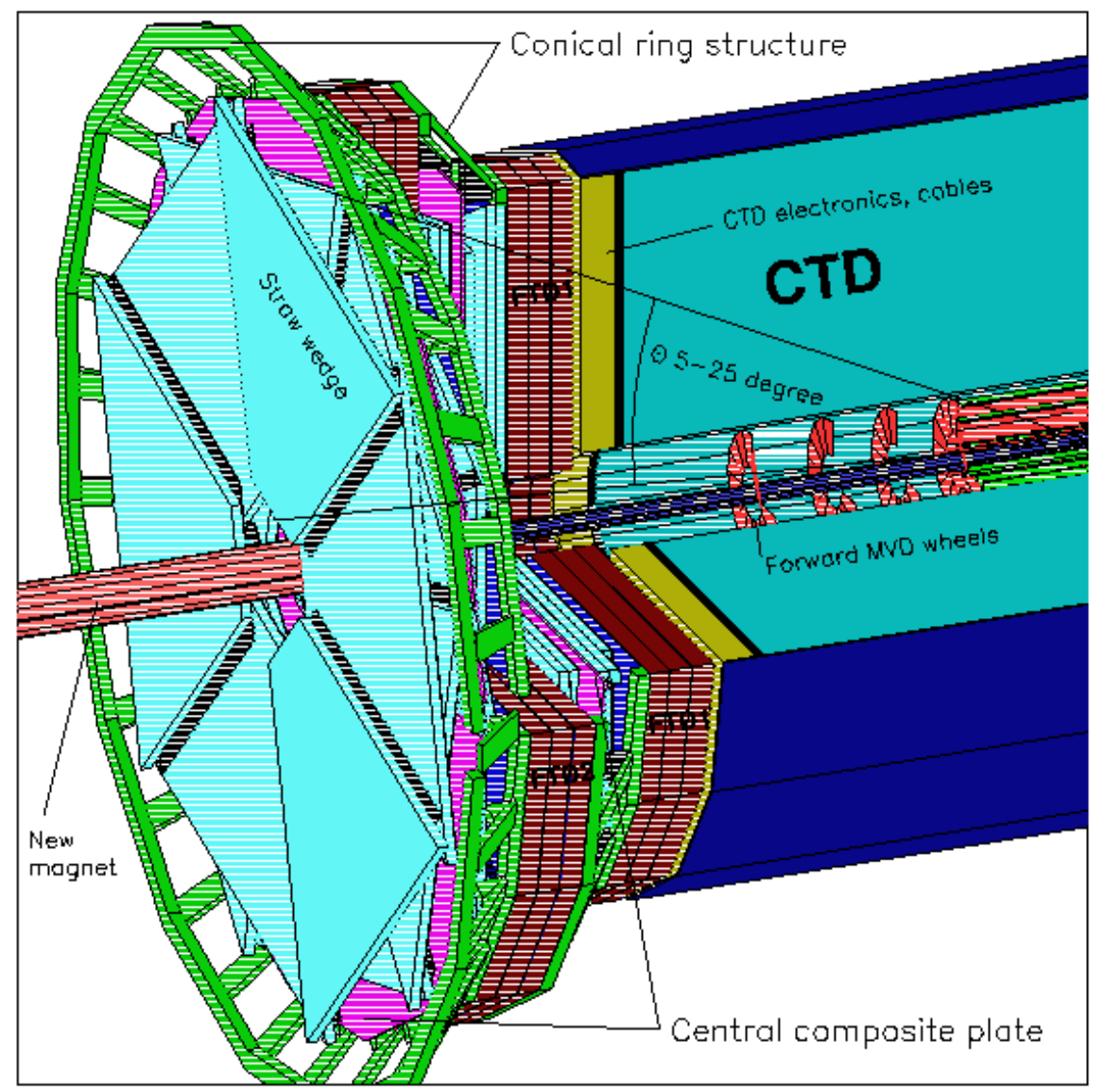

Figure 3. Schematic view of the two STT supermodules and two of the three planar drift chamber modules.

Groups of six straws are multiplexed to a single FADC channel. New front-end pre-amplification and shaping circuitry based on the ASDQ chip, as well as cable drivers, were constructed. The detector was installed in ZEUS in May, 2001.

One of the major gains in the improved forward tracking will be in the measurement of the $F_{2}$ structure function at high $Q^{2}$ and $x$. An estimate 6 


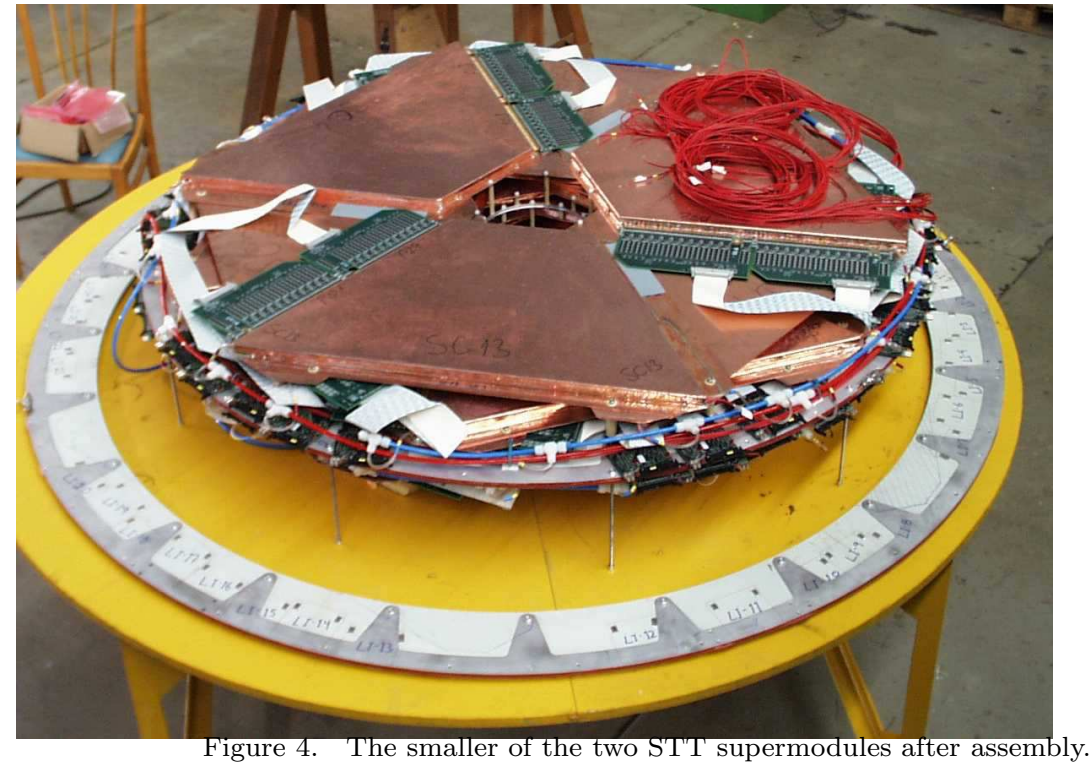

of the accuracy that can be achieved with $1 \mathrm{fb}^{-1}$ is shown in Fig. 5 , together with the corresponding accuracy of the gluon distribution function extracted from a QCD fit to this data.

\section{Luminosity monitor}

The high luminosity at HERA II together with the large synchrotron-radiation background require a new approach to the measurement of luminosity at ZEUS. The simple photon calorimeter used previously must be upgraded to cope with the background and the increased probability for multiple bremsstrahlung photons in one beam-crossing. In order to improve the accuracy of the luminosity measurement, a second technique, with very different systematic uncertainties, based on an electron-positron pair spectrometer, has been constructed. Both devices use the information from a small calorimeter placed around $6 \mathrm{~m}$ from the interaction point which detects the radiating electron. The set-up is shown schematically in Fig. 6.

The photon calorimeter is a lead-scintillator sandwich with a position detector consisting of strips of scintillator. In order to cope with the synchrotron radiation background, an "active filter", consisting of two carbon absorbers, each of two radiation lengths, alternating with Aerogel Cerenkov detectors 

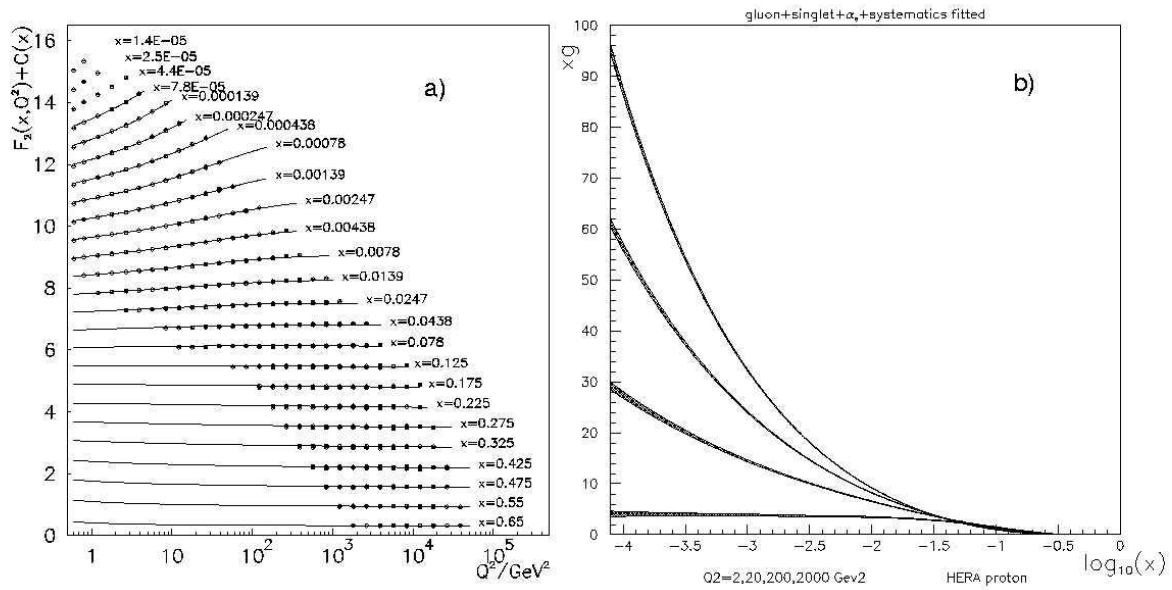

Figure 5. a) Estimate of the accuracy with which $F_{2}$ can be measured in $x$ bins as a function of $Q^{2}$ with $1 \mathrm{fb}^{-1}$ at HERA II. b) Estimate of the accuracy to which the gluon distribution function can be extracted from a QCD fit to such a data sample.

has been constructed. The absorbers protect the calorimeter from radiation damage, while the Cerenkov detectors detect high-energy photons that convert in the absorbers, allowing the calorimeter energy to be corrected and good resolution to be recovered. The calorimeter is currently being installed in the HERA tunnel.

The pair spectrometer is situated downstream of an exit window corresponding to around $12 \%$ of a radiation length. The electron-positron pairs that covert therein are separated by a dipole magnet and detected in a pair of tungsten-scintillator sandwich calorimeters, previously used to detect smallangle, very low- $Q^{2}$ electrons close to the beam-line in the main ZEUS detector. These devices have been refurbished and installed in the spectrometer in April 2001.

The " $6 \mathrm{~m}$ tagger" consists of a $10 \times 10 \times 5 \mathrm{~cm}$ tungsten-scintillating fibre calorimeter next to the beam-pipe inside one of the HERA magnets. The device has been tested in the DESY test beam and shown to be linear to better than $1 \%$, to have a relative energy resolution of $17 \% / \sqrt{E}$ and a uniformity better than $5 \%$. In order to protect against possible radiation damage, the tagger will be installed after HERA commissioning is complete.

Each of these devices uses a common electronic readout system. This will be completed, installed and tested in the next few months. It is hoped to 


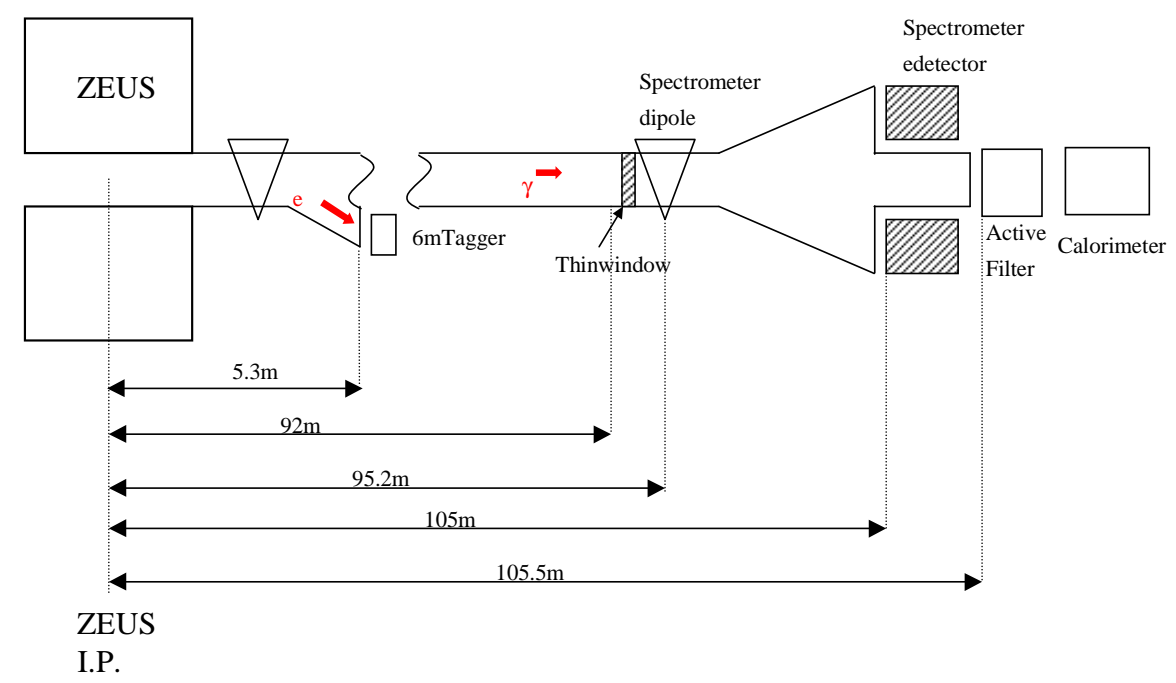

Figure 6. Schematic view of the ZEUS luminosity monitor system, consisting of an electron tagger, a pair spectrometer, and a photon calorimeter with active filter.

attain a luminosity measurement precision of around $1 \%$.

\section{Polarisation}

For several years, HERMES have trail-blazed the use of polarised electrons at HERA and, in close partnership with the HERA accelerator physicists, polarisations of around $65 \%$ have been achieved. It is hoped to increase the accuracy with which the polarisation can be measured to $\delta P / P \sim 2 \%$ per bunch per minute. This will be achieved by a collaboration between H1, HERMES, ZEUS and the HERA machine in the POL2000 project. The collaboration is constructing two instruments, both of which detect asymmetries in backscattered light from high-intensity polarised lasers. One, the LPOL, measures the energy asymmetry between left- and right-handed polarised scattered photons, which, in the photon-electron centre-of-mass frame, can be understood as arising from the relative orientation of the electron and photon spin leading to orbital angular momentum of either $1 / 2$ or $3 / 2$. When boosted back to the laboratory system, this results in a difference in energy between the two situations. It is proposed to use a Fabry-Pérot cavity in order to permit very fast bunch-by-bunch measurements. The other detector, the TPOL, measures the 

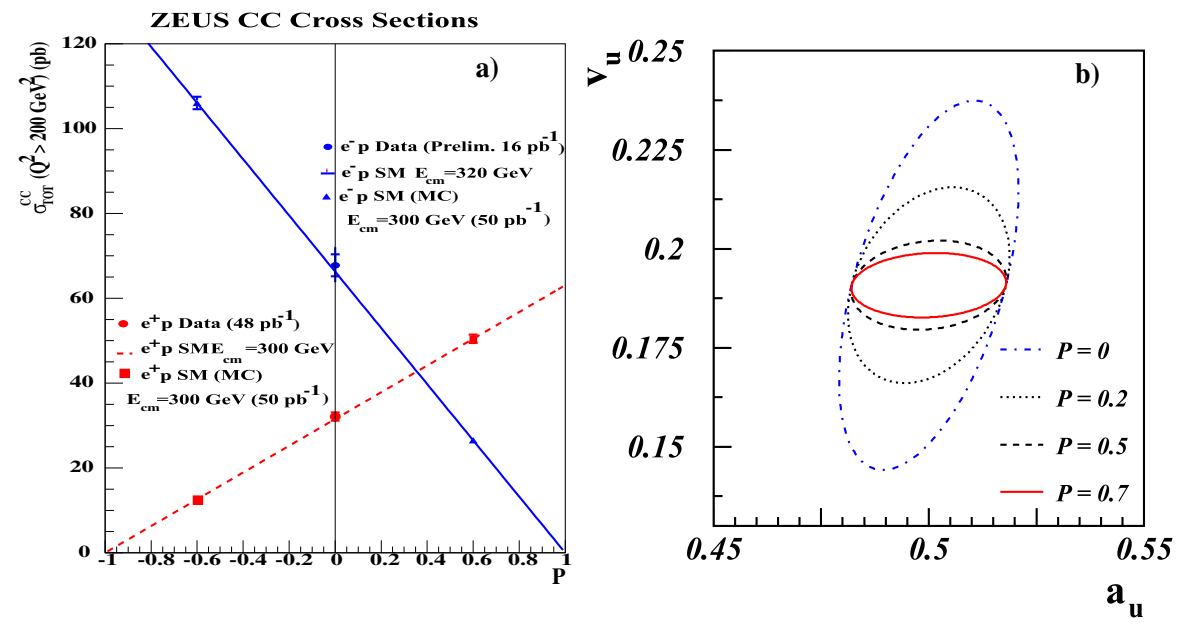

Figure 7. a) The cross section for charged current interactions. The points at $\mathrm{P}=0$ are obtained from ZEUS preliminary results at the indicated centre-of-mass energies, while those at non-zero polarisation are Monte Carlo simulations of the expected accuracy in ZEUS assuming the Standard Model cross section for an integrated luminosity of $50 \mathrm{pb}^{-1}$ per point. b) One standard-deviation contours for the $u$-quark vector and axial couplings from fits to Monte Carlo data in which the $d$-quark couplings are held constant. The various contours correspond to $1 \mathrm{fb}-1$ of data with the degrees of polarisation indicated.

degree of transverse polarisation by measuring the up-down asymmetry of the detected photons. This asymmetry arises since the transverse spin direction defines a preferred spatial axis with respect to the beam direction. The TPOL uses a high-precision position detector, which is the ZEUS contribution to the POL2000 project, plus a calorimeter to measure this asymmetry.

The combination of high-precision measurements of both luminosity and polarisation will be important in a wide range of HERA II physics. The most obvious application is in the electroweak sector, where, for example, the charged current cross section should vanish for the appropriate combinations of lepton charge and polarisation. A measurement at three polarisations, such as shown in Fig. 7la), even with relatively modest luminosity, will provide an accurate test of this prediction, and provide mass limits on right-handed $W \mathrm{~s}$ higher than $400 \mathrm{GeV}$.

Strong polarisation effects are also predicted at high $Q^{2}$ in the neutral current, where, e.g. at $Q^{2}=10^{4} \mathrm{GeV}^{2}$ and $x=0.2$, there is a factor two difference between the predicted cross sections for left- and right-handed elec- 
trons. The luminosity available at HERA II will permit the determination of the $u$ - and $d$-quark couplings. The gain in the determination of the $u$ quark electroweak couplings from $1 \mathrm{fb}^{-1}$ of simulated data $\mathrm{E}$ as the degree of polarisation is increased is clearly visible in Fig. $7 \mathrm{~b}$ ).

In addition to the use of precise luminosity and polarisation information in the study of electroweak processes, polarisation also offers an invaluable tool in the study of possible signals beyond the Standard Model. Varying the polarisation to reduce the cross sections of Standard Model processes could well improve the signal to background for new physics signals, such as leptoquarks or supersymmetric particles that violate $R$ parity, for which HERA will still have the highest sensitivity for the next few years.

\section{Summary}

The summer of 2001 will see HERA and the general-purpose experiments H1 and ZEUS upgraded and operational. The promise of the HERA II programme is great. HERA I produced deep insights into QCD and laid the foundations for the study of the space-like electroweak interaction at high $Q^{2}$. HERA II promises to build on those foundations to open new fields of precision electroweak study and searches for physics beyond the Standard Model. The prospects for the new few years are indeed exciting.

\section{References}

1. A. Garfagnini, Nucl. Instrum. Methods A435, 34 (1999).

2. K. Daum et al in Proceedings of the Workshop on Future Physics at HERA ed. A. De Roeck, G. Ingleman and R. Klanner, Vol. 1, p. 89 (DESY, Hamburg, 1996).

3. V. Barone, U. D'Alesio and M. Genovese, ibid, p. 102.

4. M. Klein, M. Botje and C. Pascaud, ibid, p. 33.

5. R.J. Cashmore et al, ibid, p. 163. 